\title{
Die sakramentale Kraft des Wassers. Scholastische Debatten über ein augustinisches Bild zur Wirkweise von Weihwasser und Taufe
}

\begin{abstract}
Whence has water so great an efficacy, as in touching the body to cleanse the soul?” This famous question from Augustine's 'Tractates on the Gospel of John' asked rhetorically with regard to Baptism - was for centuries an object of concern for medieval theologians trying to explain the efficacy of the sacramental administration of grace. In particular, the striving for rationality within late medieval scholasticism seemed to encounter insurmountable problems, for how could it be that a corporeal element such as water could bestow something as spiritual as grace? How could a natural event such as the contact with water produce a supernatural purification of the soul? Augustine's own answer, that this was due to the belief in the accompanying words, was only one of several models that late medieval scholasticism developed and transferred from the question of sacramental efficacy to related problems such as the efficacy of holy water. Starting with a late scholastic tract on holy water, the 'De efficacia aquae benedictae' of Juan de Torquemada (written ca. 1437 at the council of Basel), this contribution explores the different models of explanation for the special power of water and illustrates the role that the particular properties of water assumed for the development of a general theology of the sacraments.
\end{abstract}

Keywords: Taufe, Weihwasser, Augustinus, Juan de Torquemada

In memoriam

Urs M. Zahnd († 2014)

In den späten dreißiger Jahren des 15. Jahrhunderts verfasste Juan de Torquemada, ein spanischer Dominikaner und hoch angesehener Theologe, der als Gesandter des Königs von Kastilien am Basler Konzil mitstritt, einen kleinen Traktat über die Wirksamkeit des Weihwassers. ${ }^{1}$ Die Frage, inwiefern geweihtem Wasser eine eigene, intrinsische Wirkung zum Schutz der Gläubigen und zur Vertreibung der Dämonen innewohne, war aus scholastischer Perspektive nicht ganz unproblematisch, doch

1 ,De efficacia aquae benedictae' oder auch ,Tractatus de aqua benedicta', im Folgenden zitiert nach der Ausgabe Rom 1475. Die genaue Abfassungszeit ist unklar: Thomas KAEPPELI, Scriptores Ordinis Praedicatorum Medii Aevi, vol. III (I-S), Rom 1980, plädiert für 1438, Thomas M. IzBICKI, Protector of the Faith. Cardinal Johannes de Turrecremata and the Defense of the Institutional Church, Washington 1981, S. 128 (Anm. 24) für den Jahreswechsel 1436/1437. 
war sie nicht eigentlich eines der in Basel groß debattierten Themen. ${ }^{2}$ Torquemada, der am Konzil zu einem der eifrigsten Verfechter des päpstlichen Primats wurde und konsequent gegen jegliche Zugeständnisse an die Hussiten $\mathrm{zu}$ wirken versuchte, ${ }^{3}$ hätte denn wohl auch besseres zu tun gehabt, wäre da nicht der böhmische Gesandte gewesen, Peter Payne, ein nach Prag emigrierter Engländer, der als Verteidiger einer radikalen hussitischen Richtung nach Basel gereist war, dort aber - nicht nur zu seinem eigenen, sondern auch zum Unmut Torquemadas - die mehrheitliche Kompromissbereitschaft und ein beiderseitiges Entgegenkommen in der Hussitenfrage nicht verhindern konnte. ${ }^{4}$ Denn dieser Peter Payne scheint in einer der Debatten insbesondere um den Laienkelch das Argument angeführt zu haben, dass der traditionelle katholische Ritus, der den Laien bei der Eucharistie die Teilnahme bloß an der Brotkommunion, und nicht sub utraque specie, an Brot und Wein, gestattete, die Wirkkraft der Eucharistie unter jene von geweihtem Wasser stelle. ${ }^{5}$

Das klang auch in traditionellen katholischen Ohren zuerst einmal skandalös, galt doch die Verwendung von Weihwasser nicht einmal als Sakrament, während die Eucharistie, die Vergegenwärtigung des Opfertods Christi, das höchste der Sakramente schlechthin war. Auf Bitten des Vorsitzenden des Konzils, seines Ordensbruders Johannes de Ragusa, nahm sich Juan de Torquemada daher der Problematik an und führte in seinem Traktat unter Rückgriff auf ein ganzes Bouquet von juristischen und theologischen Quellen aus, ${ }^{6}$ dass nicht nur dem Weihwasser selbst bei seiner Anwen-

2 Locus classicus der scholastischen Diskussion über das Weihwasser waren die Kommentare zu Petrus Lombardus, Sententiae in quatour libros distinctae IV, d. 6, c. 7, hrsg. v. Ignatius BRADY (Spicilegium Bonaventurianum 5), Rom 1971/81, S. 275 f.

$3 \mathrm{Zu}$ Torquemada vgl. neben IZBICKI (Anm. 1) auch Thomas PRÜGL, Modelle konziliarer Kontroverstheologie. Johannes von Ragusa und Johannes von Torquemada, in: Heribert MüLLER u. Johannes Helmrath (Hgg.), Die Konzilien von Pisa (1409), Konstanz (1414-1418) und Basel (1431-1449). Institution und Personen (Vorträge und Forschungen 67), Ostfildern 2007, S. 257-287, bes. S. 260; Ulrich HoRST, Konziliarismus und Papalismus im Widerstreit von Juan de Torquemada bis Francisco de Vitoria, in: Bernward SchmidT u. Hubert Wolf (Hgg.), Ekklesiologische Alternativen? Monarchischer Papat und Formen kollegialer Kirchenleitung (15.-20. Jahrhundert) (Symbolische Kommunikation und gesellschaftliche Wertesysteme 42), Münster 2013, S. 55-75, hier S. 56-59.

4 Dazu William R. CooK, John Wyclif and Hussite Theology 1415-1436, in: Church History 42 (1973), S. 335-349, bes. S. 347; allgemein zu den Hussiten-Verhandlungen in Basel vgl. František ŠMAHEL, Die Hussitische Revolution (Schriften der Monumenta Germaniae Historica 43), Hannover 2002, Bd. III, S. $1560-1589$.

5 So zumindest die Informationen, die aus Torquemadas Traktat (Anm. 1) zu gewinnen sind: Fraglich geworden sei ein probleuma Reverendo magistro Iohanne de Ragusio primo Bohemorum articulo respondenti per magistrum Petrum Anglicum Prepositum [...] videlicet Utrum aqua benedicta sit maioris efficacie et virtutis quam sacramentum sensibile altaris (fol. a1v).

6 Etwas mehr noch als aus dem ,Decretum Gratiani` zitiert Torquemada aus der Bibel; fast gleich wichtig sind ihm zudem Hugo von St. Viktor und Thomas von Aquin. Darüber hinaus finden sich vereinzelte Verweise auf rund zehn weitere scholastische Quellen (Glossen zur Bibel und zum ,Decretum', Geschichtswerke, Beda Venerabilis, Bernhard von Clairvaux, Petrus Lombardus, Wilhelm 
dung tatsächlich eine eigene, intrinsische Wirksamkeit zukomme, sondern dass man durchaus mit Fug behaupten könne, diese Kraft sei größer als jene, welche in den eucharistischen speciebus, im Anschein des gewandelten Brots und des gewandelten Weins zu finden sei - denn dort sei eine solche Kraft überhaupt nicht vorhanden. ${ }^{7}$ In gewisser Hinsicht schien Torquemada seinen hussitischen Gegner damit sogar zu bestätigen, nur war dies in des Spaniers Augen der Würde der Eucharistie in keiner Weise abträglich: Anders als bei den übrigen Sakramenten lag deren Kraft und Wert gerade nicht in den äußerlichen Elementen, die nach vollzogener eucharistischer Wandlung bloß dem Ansehen nach Brot und Wein waren, ${ }^{8}$ sondern es wirkte die nicht wahrnehmbar gewandelte Substanz von Leib und Blut Christi. Damit bestätige sich gegen die Hussiten vielmehr, wie wenig man die Eucharistie sub utraque specie empfangen müsse, um ihrer vollen Wirkung teilhaftig zu werden. ${ }^{9}$

Dieser Traktat über das Weihwasser war äußerst erfolgreich. Dank der internationalen Plattform, die das Basler Konzil bot, und wohl auch dank der Tatsache, dass sein Autor zur schließlich siegreichen Seite der Papalisten gehörte, ${ }^{10}$ fand er in ganz Europa Verbreitung. Bis heute ist er in gut fünfzig Handschriften erhalten, ${ }^{11}$ noch im 15. Jahrhundert wurde er mehr als zehn Mal gedruckt und es folgten einige weitere Druckauflagen mindestens bis in die 30er Jahre des 16. Jahrhunderts hinein. ${ }^{12}$ Dieser Erfolg überrascht - nicht nur angesichts der sehr spezifischen und nebensächlichen Thematik des Traktats, sondern auch wegen des nicht unproblematisch dünnen Eises, auf das sich Torquemada begab, indem er den sichtbaren Elementen der Eucharistie eine kleinere Wirksamkeit zusprach als dem Weihwasser und dafür

Duranti, Albertus Magnus, Petrus de Palude und Durandus von St. Pourçain) sowie auf die kirchliche liturgische Tradition. Zu Torquemadas Autoritätengebrauch vgl. auch PRÜGL (Anm. 3), S. 268-272.

7 Juan de Torquemada (Anm. 1), fol. c6v: Patet probleumatis solutio quod in aqua benedicta modo quo supra dictum est sit aliqua virtus, sit aliqua efficatia ad effectus supra notatos, et quod in speciebus sacramentalibus secundum se consideratis circumscripto corpore Christi vero nulla sit virtus, nulla efficatia ad aliquem effectum causandum in ipsa anima.

8 Ebd., fol. c4v: Species predicte sacramentales secundum se, hoc est circumscripto corpore Christi, vero nullam habeat virtutem spiritualem nec efficatiam causalitatis cuiuscunque effectus in animo sus[cip]ientis sacramentum.

9 Ebd., fol. c6v: Ex quo patet correlarie quod ex opere operato sive ex parte ipsius sacramenti non est maior virtus huius sacramenti sanctissimi altaris sive efficaci[a] sub duabus speci[e]bus quam sub una specie, cum illa res et radix totius efficacie huius sacramenti non sit maior, non plenior, non perfectior sub duabus speciebus quam sub una.

10 Vgl. PRÜGL (Anm. 3), S. 260 f.

11 Grösstenteils verzeichnet bei KAEPPELI (Anm. 1), S. 31 f.; zusätzlich zu nennen sind die Handschriften Krakau, Jagiellonische Bibliothek, Cod. 1240, fols. 96v-99v; Michaelbeuern, Stiftsbibliothek, man. cart. 69, fols. 189v-194v; München, Universitätsbibliothek, 4 cod. 33, fols. 106r-116v; Stuttgart, Württembergische Landesbibliothek, HB I 223; Venedig, Biblioteca dei Redentoristi 12, fols. 73ra-76ra.

12 Der früheste Druck ist nicht, wie bei KAEPPELI (Anm. 1), S. 32, vermerkt, Rom: Bartholomaeus Guldinbeck, 1475, sondern Rom: Antonius und Raphael de Volterra, ca. 1473/74. Der späteste frühneuzeitliche Druck scheint Krakau: Hieronymus Vietor, 1533 zu sein. 
sogar in Kauf nahm, dass er das Verständnis der Wirkweise der Eucharistie von jenem der übrigen Sakramente abkoppeln musste. ${ }^{13}$ Ohnehin war diese Wirksamkeitsfrage selbst nicht unproblematisch, rüttelte sie doch ganz grundlegend an den vorherrschenden, hierarchisch-kosmologischen Vorstellungen, weil sie unterstellte, dass die irdisch-körperlichen sakramentalen Elemente eine Wirkung auf die über-natürliche, dem irdischen Zugriff sonst entzogene Gnadenwirkung haben sollten. Wurde damit nicht die Ordnung zwischen Geschöpflichem und Ungeschöpflichem auf den Kopf gestellt, wurde nicht sogar die göttliche Freiheit ungebührlich eingeschränkt, da Gott doch seine Gnade nicht in Abhängigkeit von der Wirkung irdischer Elemente spendete? ${ }^{14}$ Und auch wenn man sich in der Jahrhunderte alten scholastischen Diskussion, die dem Basler Konzil vorausging, unter Entwicklung ganz unterschiedlicher Modelle darauf eingelassen hatte, dass bei den sieben traditionellen Sakramenten eine solche Wirksamkeit allenfalls denkbar sei, liessen sich diese Überlegungen und Modelle dann so einfach auf das Weihwasser übertragen, nur weil dies als antihussitisches Argument von einigem Wert sein konnte ${ }^{15}$ Wie sollte das gehen, dass dem geweihten Wasser eine solch große, die Ebene des Körperlichen übersteigende Wirkmacht zukomme?

Es ist dies eine Frage, die mit Blick nicht auf das Weihwasser, sondern auf das Taufwasser bereits tausend Jahre früher kein geringerer als Augustinus gestellt hatte, der mit solchem Fragen nun aber das ganze mittelalterliche Nachdenken nicht nur über Taufe und Weihwasser, sondern über die Sakramente schlechthin bestimmen und damit letztendlich auch die Debatte am Basler Konzil vorentscheiden sollte. Im Folgenden soll es darum gehen, diese Entwicklung nachzuzeichnen und aufzuzeigen, wie aus dem fragendem Blick des Augustinus auf das Wasser und die in ihm liegende Kraft über einige Exponenten vor allem der spätmittelalterlichen Scholastik hinweg bis hin zu Juan de Torquemada und seinem Traktat zum Weihwasser die mittelalterliche Vorstellung über das Wesen der Sakramente und Sakramentalien entscheidend von der Symbolik des Wassers geprägt war.

Augustinus hatte sich in seinem Traktat über das Johannesevangelium staunend gefragt, woher diese so große Kraft im Wasser komme, dass es zwar bloß den Körper

13 Juan de Torquemada (Anm. 1), fol. c6r: In hoc sacramento est fontana virtus, scilicet Christus Ihesus, ergo preter ipsum Christum contentum sub illis speciebus non est dicenda aliqua virtus particularis: illa enim esset superflua. [...] In aliis autem sacramentis ubi non est hoc modo presens id quod per essentiam est fontana virtus sunt necessarie quedam participate particulares virtutes.

14 Zum Problemfeld der sakramentalen Wirksamkeit vgl. Irène RosIER-CATACH, La parole efficace. Signe, rituel, sacré, Paris 2004; Ueli ZAHND, Wirksame Zeichen? Sakramentenlehre und Semiotik in der Scholastik des ausgehenden Mittelalters (Spätmittelalter, Humanismus, Reformation 80), Tübingen 2014.

15 In umgekehrter Richtung zu argumentieren war hingegen unproblematisch, vgl. Guido Briansonis, Collectarium super sententias, Paris 1512: Sicut aqua benedicta virtute benedictionis habet vim terrendi daemones, et alias multas operationes, sic sacramenta (q.1, c.2.1, fol. 8vb). 
berühre, aber die Seele reinige. ${ }^{16}$ Er formulierte dieses Staunen über das Wasser anlässlich der Auslegung von Johannes 15,3, wo Jesus zu seinen Jüngern sagte: „Ihr seid schon rein um des Wortes willen, das ich zu euch gesprochen habe." Warum, so fragte sich Augustinus, war hier vom reinigenden Wort, und nicht etwa von der reinigenden Taufe die Rede? Das konnte, so gab er gleich zur Antwort, nur daran liegen, dass auch bei der Taufe - und entsprechend beim Taufwasser - die eigentliche Reinigung durch das Wort vollzogen werde: Denn nehme man vom Taufwasser das Wort weg, so verbleibe nichts als Wasser; komme aber zum Element das Wort hinzu, dann werde es zum Sakrament und das Wasser zu einer Art sichtbarem Wort:

Warum sagt er nicht: ,Ihr seid rein wegen der Taufe, mit der ihr gewaschen worden seid‘, sondern sagt: ,um des Wortes willen, das ich zu euch gesprochen habe', außer weil auch im Wasser das Wort reinigt? Nimm das Wort weg, und was ist das Wasser als eben Wasser? Es tritt das Wort zum Element und es wird Sakrament, auch dieses gleichsam ein sichtbares Wort. ${ }^{17}$

Für Augustinus war damit klar, dass der wesentliche Bestandteil an der Taufe nicht das Wasser, sondern das hinzukommende Wort sei, ${ }^{18}$ und wenn er im Anschluss daran nun seine erstaunte Frage nach der Kraft im Wasser stellte, so beantwortete er sie auch gleich in eben diesem Sinne, dass es am Wort liege, und zwar nicht einfach am gesprochenen Wort, sondern am Wort, dem geglaubt werde:

Woher kommt diese so große Kraft des Wassers, dass es den Leib berührt und das Herz abwäscht, außer durch die Wirksamkeit des Wortes, nicht weil es gesprochen, sondern weil es geglaubt wird? Denn auch im Wort selbst ist etwas anderes der vorübergehende Klang und etwas anderes die bleibende Kraft. ${ }^{19}$

16 Augustinus, In Iohannis Evangelium tractatus CXXIV, t. 80, n. 3, hrsg. v. Radbodus WiLLEMs (Corpus Christianorum, Series Latina 36), Turnhout 1954, S. 529: Unde ista tanta virtus aquae, ut corpus tangat et cor abluat?

17 Ebd.: ,Iam vos mundi estis propter verbum quod locutus sum vobis. 'Quare non ait, ,mundi estis propter baptismum quo loti estis', sed ait: ,propter verbum quod locutus sum vobis', nisi quia et in aqua verbum mundat? Detrahe verbum, et quid est aqua nisi aqua? Accedit verbum ad elementum, et fit sacramentum, etiam ipsum tamquam visibile verbum. Nam et hoc utique dixerat, quando pedes discipulis lavit: ,Qui lotus est, non indiget nisi ut pedes lavet, sed est mundus totus ' (Joh 13,10).

18 Was sich zum klassischen Verständnis eines Sakraments als Zusammenkommen von elementum und verbum verdichten sollte, eine Bestimmung, die sich allerdings nicht ohne weiteres auf alle Sakramente übertragen liess, vgl. die reiche Materialsammlung bei Damien vAN DEN EYNDE, The Theory of the Composition of the Sacraments in Early Scholasticism (1125-1240), in: Franciscan Studies 11 (1951), S. 1-20, 117-144, 12 (1952), S. 1-26.

19 Augustinus (Anm. 16), S. 529: Unde ista tanta virtus aquae, ut corpus tangat et cor abluat, nisi faciente verbo, non quia dicitur, sed quia creditur? Nam et in ipso verbo, aliud est sonus transiens, aliud virtus manens. Zum Sakramentenverständnis des Augustinus s. Philip CARY, Outward Signs. The Powerlessness of External Things in Augustine’s Thought, Oxford 2008. 
Unbedacht seines spezifischen exegetischen Kontexts sollte Augustinus' bildhafter Vergleich von äußerlich reinigendem Wasser und innerlich gewaschener Seele dank seiner Einprägsamkeit eine breite Rezeption erfahren, eine Rezeption allerdings, in der das Bild selbst so tragend wurde, dass in den Hintergrund trat, dass es Augustinus ursprünglich nicht nur als Frage vorgestellt, sondern auch mit einer Antwort versehen hatte. Als in den ersten großen systematischen Entwürfen der mittelalterlichen Scholastik im 12. Jahrhundert Väterzitate einzeln zusammengetragen und thematisch zu einem Gesamtbild der christlichen Lehre zusammengestellt wurden, ${ }^{20}$ erhielt das Bild vom Abwaschen der Sünde in seiner augustinischen Formulierung einen festen Platz nicht nur in der Lehre von der Taufe, sondern von den Sakramenten im Allgemeinen.

In seiner großen Gesamtschau ,Über die Heiltümer des christlichen Glauben` (De sacramentis christianae fidei) erhob Hugo von St. Viktor - weil das, was in der Taufe äußerlich vollzogen wurde, so ähnlich mit dem war, was als ihre innerliche Wirkung galt, - die Ähnlichkeit von sakramentalem Ritus und sakramentaler Wirkung zu einer allgemeinen Bestimmung dessen, was ein Sakrament sei; ${ }^{21}$ ja, mehr noch, dank dieser Ähnlichkeit habe ein Sakrament die Möglichkeit, äußerlich zu repräsentieren, was innerlich vollzogen werde, und den Menschen damit zu belehren, was das sakramentale Geschehen sei. ${ }^{22}$ Als Illustration dieses Wesens der Sakramente griff Hugo - nicht überraschend - das Beispiel des Wassers der Taufe auf: Das Wasser sei sichtbares Sakrament, und die Gnade die unsichtbare Sache oder die Kraft des Sakramentes. ${ }^{23}$ Diese Ähnlichkeit machte das Wasser nun fast schon zu einem natürlichen Zeichen dessen, was sich im Sakrament vollzog. Hugo führte aus:

20 Dazu Stéphane GioAnNi, Un florilège augustinien sur la connaissance sacramentelle. Une source de Bérenger de Tours et d'Yves de Chartres?, in: Monique Goullet (Hg.), Parva pro magnis mundera. Études de littérature tardo-antique et médiévale offertes à François Dolbeau par ses élèves (Instrumenta patristica et mediaevalia 51), Turnhout 2009, S. 699-723.

21 Debet enim omne sacramentum similitudinem quandam habere ad ipsam rem cuius sacramentum est, secundum quam habile sit ad eandem rem suam representandam: Hugo von Sankt Viktor, De sacramentis Christianae fidei I.9.2, hrsg. v. Rainer BERNDT, Münster 2008, S. 210. Ausführlich zur similitudo auch RosiER-CATACH (Anm. 14), S. 66 f.; zusätzlich zur similitudo kommen bei Hugo als entscheidende Merkmale für ein Sakrament noch die institutio und die sanctificatio hinzu, vgl. Claus BLESSING, Sacramenta, in quibus principaliter salus constat. Taufe, Firmung und Eucharistie bei Hugo von St. Viktor, Diss. Wien 2009, S. 94.

22 Hugo von St. Viktor (Anm. 21), I.9.3, S. 212: Propter eruditionem quoque instituta sunt sacramenta ut per id quod foris in sacramento in specie visibili cernitur, ad invisibilem virtutem que intus in re sacramenti constat cognoscendam mens humana erudiatur; vgl. auch ebd., I.10.2, S. 224.

23 Ebd., I.9.2, S. 210: Aquam baptismi per exemplo assumimus: ibi enim est aqua visibile elementum quod est sacramentum. [...] Est ergo aqua visibile sacramentum et gratia invisibilis res sive virtus sacramenti. 
Es hat aber jedes Wasser aus natürlicher Beschaffenheit eine gewisse Ähnlichkeit mit der Gnade des Heiligen Geistes, weil nämlich, so wie dieses den Schmutz der Leiber abwäscht, jene die Verunreinigungen der Seele abwäscht. Und aus dieser ja doch mitgegebenen Beschaffenheit vermochte jedes Wasser eine geistliche Gnade zu repräsentieren. ${ }^{24}$

Entscheidend war nun aber, dass Hugo nicht nur einfach über das Taufwasser sprach, sondern das Taufwasser als Modellfall für die Sakramente im Allgemeinen nahm und entsprechend am Ende seines Beispiels ausführte: „Nach dieser Weise ist es erforderlich, auch in den übrigen Sakramenten diese Dinge zu erwägen. “25 Wie das im Einzelnen gehen solle, führte Hugo nicht aus, aber die Symbolik lag so auf der Hand, dass in Hugos direkter Nachfolge die Behauptung gerne aufgegriffen wurde, es hätten die sakramentalen Elemente eine Art natürlich repräsentierende Funktion dessen, was im Sakrament vonstattenging. ${ }^{26}$

Einer, der diesen Ähnlichkeitsanspruch besonders prominent übernahm, war Petrus Lombardus, der Verfasser jener berühmten Sammlung von KirchenväterZitaten, den ,Sententiae libri quatuor‘ aus der Mitte des 12. Jahrhunderts, die für die weitere mittelalterliche Scholastik - und darüber hinaus ${ }^{27}$ - $\mathrm{zu}$ einem Basistext des universitären Theologiestudiums werden sollten. Petrus Lombardus trug im vierten Buch seiner Sentenzen, das den Sakramenten gewidmet war, deren repräsentierender Funktion als entscheidender Bestimmung Rechnung und definierte entsprechend ein Sakrament als „,solcherart ein Zeichen der Gnade Gottes und eine Form der unsichtbaren Gnade, dass es deren Bild hervorruft und als deren Ursache fungiert“. ${ }^{28}$ Damit ging Petrus Lombardus allerdings noch einmal über Hugo von St. Viktor hinaus. Denn nicht nur evozierte ein Sakrament - dank seiner Ähnlichkeit - ein Bild von der unsichtbaren Gnade, sondern es wurde sogar als deren Ursache definiert. Ganz im Sinne der augustinischen Frage nach der Kraft des Wassers, das die Seele reinzuwaschen vermag (aber nicht eigentlich im Sinne der augustinischen Antwort), behauptete Petrus Lombardus nun auch einen kausalen Zusammenhang zwischen

24 Ebd.: Habet autem omnis aqua ex nat[ur] ali qualitate similitudinem quandam cum gratia sancti spiritus quia sicut hec abluit sordes corporum, ita illa mundat inquinamenta animarum. Et ex hac quidem ingenita qualitate omnis aqua spiritalem gratiam representare habuit.

25 Ebd., S. 211: Ad hunc modum in ceteris quoque sacramentis tria hec considerare oportet.

26 Vgl. RosieR-CATACH (Anm. 14), S. 57-65.

27 Vgl. jüngst Lidia Lanza u. Marco Toste, The Sentences in Sixteenth-Century Iberian Scholasticism, in: Philipp W. Rosemann (Hg.), Mediaeval Commentaries on the Sentences of Peter Lombard 3, Leiden 2015, S. 415-502. Allgemein zur Sentenzentradition vgl. Philipp W. RosemanN, The Story of a Great Medieval Book. Peter Lombard's Sentences (Rethinking the Middle Ages 2), Peterborough 2007. 28 Petrus Lombardus (Anm. 2), IV d. 1, c. 4, S. 233: Sacramentum enim proprie dicitur, quod ita signum est gratiae Dei et invisibilis gratiae forma, ut ipsius imaginem gerat et causa existat. Zu dieser Definition und ihren Quellen vgl. bereits Damien VAN DEN EYNDE, Les définitions des sacrements pendant la première période de la théologie scolastique (1050-1240), Rom 1950 und nun auch RosIER-CATACH (Anm. 14), S. 96-98. Zum Folgenden s. ZAHND (Anm. 14), S. 126. 
Beschaffenheit der sakramentalen Elemente und der durch die Sakramente initiierten Gnadenwirkung. Erneut scheint dafür die Symbolik des Wassers Pate gestanden zu haben, ${ }^{29}$ und damit war in der scholastischen Diskussion die Bestimmung verankert, dass den Sakramenten - damit sie als echte Ursachen der Gnade gelten konnten - eine eigene, intrinsische Kraft in der Bewirkung des sakramentalen Effekts zukommen müsse.

In der nachfolgenden Diskussion führte dies zu großen Debatten darüber, wie eine solche Wirkung zu verstehen sei, damit sie eben die Ordnung zwischen natürlichem Bereich, zu dem die sakramentalen Elemente und auch das Wasser gehörten, und übernatürlichem Bereich, zu dem die Gnade und ihre Wirkung gehörten, nicht in Frage stellte. Das wirkmächtigste Modell einer Verteidigung eines tatsächlichen, intrinsischen sakramentalen Effekts entwarf dabei zweifelsohne Thomas von Aquin in einem Kommentar zu den Sentenzen des Petrus Lombardus (vor 1256). ${ }^{30}$ Ausgehend von einer gekürzten Version des Augustinus-Zitats, der er entnahm, dass die Sakramente die Gnade tatsächlich verursachen würden, ${ }^{31}$ hielt er zuerst einmal fest, dass wegen der Autorität unter anderem des Augustinus alle gezwungen seien, die Sakramente für echte Ursachen der Gnade zu halten. ${ }^{32}$ Um nun die Schwierigkeiten zu umgehen, die mit einer direkten Wirkung der natürlichen Sakramente auf die übernatürliche Gnade verbunden waren, führte er das Konzept einer ,instrumentalen“ Ursächlichkeit in die Sakramentendiskussion ein: Zwar wirkten die Sakramente nicht selbst, sondern seien bloss als Werkzeuge (qua Instrumente) der göttlichen Gnade am Gnadenvollzug beteiligt, doch blieben sie damit ursächlich ins Gnadengeschehen involviert, weil sie als Werkzeuge benutzt würden, während die eigentliche Handlung aber dem übernatürlichen, hauptsächlichen Akteur - nämlich Gott - vorbehalten sei. ${ }^{33}$ Pate stand zur Erläuterung dieses Wirksamkeitsmodus einmal mehr das Bild des Wassers:

$29 \mathrm{Zu}$ seiner Rezeption des augustinischen Wasservergleichs s. Petrus Lombardus (Anm. 2), IV d. 3, c. 1, S. 243.

30 Die etwas modifizierte Theorie, die Thomas in seiner späteren Summa Theologiae entwarf, sollte erst in der Frühen Neuzeit rezipiert werden, dazu Klaus HEDwig, Efficiunt quod figurant. Die Sakramente im Kontext von Natur, Zeichen und Heil (S.th. III, qq. 60-65 und q. 75), in: Andreas SPEER (Hg.), Thomas von Aquin: die summa theologiae, Berlin 2005, S. 401-425, hier S. $403 \mathrm{f}$.

31 Augustinus dicit:,Quae est vis aquae ut corpus tangat et cor abluat?' Ergo habet aliquam virtutem (Thomas von Aquin, Scriptum super Sententiis, IV d. 1, q. 1, a. 4, qc. 2, n. 108, hrsg. v. Maria Fabianus Moos, Rom 1947, S. 28).

32 Ebd., n. 138, S. 34: Propter auctoritates inductas necesse est ponere aliquam virtutem supernaturalem in sacramentis. Vgl. ebd., n. 119, S. 31: Omnes coguntur ponere sacramenta novae legis aliquo modo causas gratiae esse, propter auctoritates quae hoc expresse dicunt.

33 Ebd., n. 124-127, S. 32: Sciendum quod causa efficiens [...] potest dividi [...] in agens principale, et instrumentale. Agens enim principale est primum movens, agens autem instrumentale est movens motum. Instrumento autem competit duplex actio: una quam habet ex propria natura, alia quam habet prout motum a primo agente. [...] Dicendum est ergo quod principale agens respectu justificationis Deus est, 
Solcherart materiellen Werkzeugen kommt nämlich eine gewisse Tätigkeit gemäss ihrer eigenen Natur zu, so wie dem Wasser das Abwaschen oder dem Öl das Geschmeidigmachen des Körpers. Aber darüber hinaus, sofern sie Werkzeuge der rechtfertigenden göttlichen Barmherzigkeit sind, gereichen sie bloß instrumentell zu einer bestimmten Wirkung in der Seele. Und das ist es, was Augustinus sagt, dass das Taufwasser den Körper berührt und das Herz reinwäscht. ${ }^{34}$

Mithilfe der Symbolik des Wassers gelang es Thomas, sogar einen Lösungsvorschlag für die Ursächlichkeit der Sakramente zu entwickeln - doch so sehr auch dieses Modell ganz grundlegend von der Symbolik des Wassers geprägt war, die in erster Linie die Taufe betraf, hielt auch Thomas selbstverständlich dafür, dass es für die Sakramente im Allgemeinen galt und die Idee einer instrumentalen Ursächlichkeit auch auf die übrigen Sakramente zu übertragen sei. ${ }^{35}$

Nun blieb die Behauptung einer eigenen Kausalität der Sakramente nicht unhinterfragt. Der historisch wirkmächtigste Gegenentwurf zu Thomas stammte zweifelsohne aus der Feder von Johannes Duns Scotus, ${ }^{36}$ doch wurde schon viel früher, sogar noch vor Thomas’ Ausformulierung des ,Mitwirkungs-Modells‘ Kritik an jeder Form von sakramentaler Ursächlichkeit laut. Einer der interessantesten frühen Kritiker ist der englische Dominikaner Richard Fishacre, ${ }^{37}$ der ebenfalls in einem Kommentar zu den Sentenzen des Petrus Lombardus kurz nach 1240 seinem Unmut über die ,Mitwirkungs-Theoretiker‘ Luft machte. Nach einer Auflistung von nicht weniger als elf Argumenten gegen eine eigene Wirksamkeit der Sakramente ${ }^{38}$ hielt er entnervt fest, dass er entweder die Magister nicht verstehe, oder aber mehreren von ihnen ein Fehler unterlaufen sei. ${ }^{39}$ Und um zu zeigen, wo dieser Fehler liege, griff er einmal mehr auf Beispiele mit Wasser zurück:

nec indiget ad hoc aliquibus instrumentis ex parte sua; sed propter congruitatem ex parte hominis justificandi [...] utitur sacramentis quasi quibusdam instrumentis justificationis. Für einen Überblick über die ausführliche Literatur zu Thomas' Modell der sakramentalen Wirkweise vgl. ZAHND (Anm. 14), S. 150.

34 Thomas von Aquin (Anm. 31), n. 127-130, S. 32f.: Hujusmodi autem materialibus instrumentis competit aliqua actio ex natura propria, sicut aquae abluere et oleo facere nitidum corpus; sed ulterius inquantum sunt instrumenta divinae misericordiae justificantis, pertingunt instrumentaliter ad aliquem effectum in ipsa anima. [...] Et hoc est quod Augustinus dicit quod aqua baptismi ,corpus tangit et cor abluit'.

35 Vgl. die im unmittelbaren Anschluss an das vorherige Zitat gezogene allgemeine Folgerung: Et ideo dicitur quod sacramenta efficiunt quod figurant.

36 Dazu Rosier-CATACH (Anm. 14), S. 140-156; ZAHnd (Anm. 14), S. 167-191.

37 Zu Fishacre vgl. James R. Long u. Maura O'CARRoLl, The Life and Works of Richard Fishacre OP. Prolegomena to the Edition of his Commentary on the Sentences (Bayerische Akademie der Wissenschaften. Veröffentlichungen der Kommission für die Herausgabe ungedruckter Texte aus der mittelalterlichen Geisteswelt 21), München 1999.

38 Dazu ZAHND (Anm. 14), S. 133.

39 Richard Fishacre, In IV libros sententiarum, d. 1, ad 6, q. 2, hrsg. v. Joseph GoERING, München i. E., S. 44: Aut magistros non intelligo, aut plures falsum hic habent in manibus, aestimantes aliqui unum 
Wenn dir Gott eines Tages sagen würde: ,Erlaube Dir, eingetaucht zu werden, und wenn Du die und die Worte aussprichst, werde ich dich von deiner Krankheit heilen', und du machst dies und wirst darauf geheilt, dann kannst Du sowohl sagen, dass du durch das Wasser und die und die Worte geheilt worden bist, als auch durch Gott; aber das eigentlich Bewirkende der Gesundheit war Gott, und das Wasser und die Worte waren bloß etwas, ohne die es Gott nicht gemacht hätte. ${ }^{40}$

Was Fishacre damit ins Spiel brachte, war die Idee einer causa sine qua non, einer Bedingung, ohne deren Erfüllung gewisse Wirkungen nicht zustande kamen, die aber deswegen dennoch nicht im eigentlichen Sinne ursächlich ins Geschehen involviert war. ${ }^{41}$ Ähnlich, so Fishacre, sei auch eine Geschichte aus 1 Sam 5 zu verstehen, in der Naaman, nachdem er siebenmal in den Jordan eingetaucht sei, auf das Wort des Propheten hin von der Lepra geheilt wurde: Auch Naaman könne sagen, dass ihn das Wasser geheilt habe oder der Prophet oder, im wahrsten Sinne, dass ihn Gott auf das Wort des Propheten hin durch das Wasser geheilt habe. So taufe und rechtfertige im eigentlichsten Sinne Gott, in einem übertragenen Sinne auch das durchs Wort geheiligte Wasser und der Priester. ${ }^{42}$ Im Wissen um die intuitiv einleuchtende Symbolik des Wassers bemühte sich Fishacre, mit alternativen Wasserbeispielen von der Idee wegzukommen, dass das Wasser selbst eine Wirkung habe, und er versuchte dadurch, den ,Mitwirkungs-Modellen` ein entscheidendes Element ihrer Überzeugungskraft zu nehmen.

Die Frage blieb allerdings, wie dann mit der Autorität eines Augustinus umzugehen sei, der doch wohl kaum bloße causae sine quibus non im Sinn gehabt haben konnte oder in übertragender Rede gesprochen hatte. Noch der genannte Franziskaner Johannes Duns Scotus mühte sich am Beginn des 14. Jahrhunderts mit dieser Autoritätenproblematik ab, weil ja Augustinus eindeutig von einer Kraft des Wassers gesprochen habe, ${ }^{43}$ und es sollte noch fast eine Generation dauern, bis ein weite-

aliquid esse ex verbo et aqua in baptismo, et verbum esse formam et aquam materiam, et hoc coniunctum vere habere rationem efficientis immediati respectu iustificationis in anima, Deum vero agentem mediatum. Et alii, qui non hoc de verbo, sed potius dicunt aliquid divinum a verbo vocali fieri in aqua, et hoc esse formam, et similiter unum efficere cum aqua, et communiter ab utroque esse sanctificationem. Quod quomodo stare possit, vel hoc vel illud non video.

40 Ebd.: Si diceret tibi Deus quacumque die sic: ,Te permiseris immergi, et cum tali verborum prolatione sanabo te ab infirmitate tua', si hoc faciens, deinde curareris, et dicere posses te curatum per aquam et verba talia, et per Deum, et proprie efficiens sanitatis fuisset Deus, sed aqua et verbum sunt sine quibus non fecit.

$41 \mathrm{Zu}$ den causae sine quibus non vgl. William J. Courtenay, The King and the Leaden Coin. The Economic Background of ,sine qua non' causality, in: Traditio 28 (1972), S. 185-209.

42 Richard Fishacre (Anm. 39), S. 44: Sic Naaman, septimo mersus in Iordane, quod et typum gessit baptismi, ad verbum prophetae sanatus est a corporali lepra, et dici poterat quod eum sanasset aqua. Item, quod propheta. Item, verissime, quod Deus ad verbum prophetae per aquam. Sic propriissime Deus baptizat et iustificat, per extensionem sermonis, aqua sanctificata verbo et sacerdos.

43 Johannes Duns Scotus, Ordinatio, IV d. 1, p. 3, q. 1-2, n. 324, hrsg. v. Joseph R. Carballo, Rom 2008, S. 115. 
rer Franziskaner, Petrus Aureoli, zu Augustinus zurückkehrte und sich dessen Text genauer vornahm. Was er dort fand, ermutigte Petrus Aureoli, der die große Inszenierung nicht scheute, erneut in einem Kommentar zu Petrus Lombardus Sentenzen (ca. 1317) theatralisch auszurufen:

Ich, ich habe bis zu diesem Zeitpunkt nicht eine einzige autoritative Stelle irgendeines Heiligen gesehen, welche eher die Schlussfolgerung dieses [Mitwirkungs]-Modells auszudrücken scheint als dessen Gegenteil, weshalb ich mich diesem Modell nicht anschließe, was ich umgehend tun würde, wenn ich hierfür die kleinste Autorität finden würde. ${ }^{44}$

Angesichts des bisherigen Verlaufs der Debatte musste diese Ankündigung mehr als erstaunen, doch Petrus Aureoli blieb einen Beweis nicht schuldig und zitierte umgehend die fragliche Augustinus-Stelle in ihrem Kontext, so dass er Augustinus' eigene Antwort auf die Frage nach der Kraft im Wasser wieder stark machen und damit aufzeigen konnte, dass auch Augustinus selbst diese Kraft nicht im Wasser, sondern im zugehörigen geglaubten Wort lokalisiert hatte. ${ }^{45}$

Für die unmittelbare Diskussion der Frage nach der sakramentalen Wirkweise hatten diese Einwände und Klärungen zur Folge, dass im weiteren Verlauf des 14. Jahrhunderts Verteidiger des ,Mitwirkungs-Modells‘ zu einer fast vernachlässigbaren Minderheit wurden. ${ }^{46}$ Allein, die Wassersymbolik hatte ja längst nicht mehr nur die Wirksamkeitsfrage, sondern die grundsätzliche Bestimmung, das grundsätzliche Verständnis dessen, was ein Sakrament sei, geprägt; und so sehr sich daher auch an der Oberfläche der Sakramentenlehre einige Verschiebungen vollzogen, hatte die Symbolik des Wassers ihre Kraft längst so weit ausgebildet, dass an die daran anknüp-

44 Petrus Aureoli, Commentaria super sententiarum libros, IV, d. 1, q. 1, a. 1, Rom 1605, S. 9bC: Ego autem non vidi adhuc auctoritatem aliquam alicuius sancti quae magis videatur exprimere conclusionem istius positionis quam eius oppositum, propter quod opinionem non teneo, quam tenerem utique si ad hoc auctoritatem minimam reperirem.

45 Ebd., S. 10aA-B: Capio auctoritates, quas maxime opinio ista pro se allegat, et una quidem est Augustini super Ioannem [...]. Ecce quod circumstantia expressa verborum huius auctoritatis ipsa ostendit oppositum eius, quod opinio intendebat, videlicet quod virtus sacramenti cor abluens non sit virtus inhaerens sacramentis [...]. Hoc quod dicit manens, est contra opinionem dupliciter. Primo, quia opinio ponit qualitatem illam et virtutem esse in sacramentis tantum in fluxu et in fieri. Sed auctoritas dicit virtus manens. Secundo, quia isti ponunt virtutem illam formaliter inhaerere sacramentis. Ergo oportet quod transeat transeunte sacramento. Sed auctoritas dicit quod transeunte sono remanet virtus manens. Vgl. Lauge Olaf NiELSEn, Signification, Likeness, and Causality. The Sacraments as Signs by Divine Imposition in John Duns Scotus, Durand of St. Pourçain, and Peter Auriol, in: Constantino MARMo (Hg.), Vestigia, imagines, verba. Semiotics and Logic in Medieval Theological Texts (XIIth-XIVth Century) (Semiotic and Cognitive Studies 4), Turnhout 1997, S. 223-253.

46 Für die wenigen bekannten Beispiele vgl. Ueli ZAHND, Plagiats individualisés et stratégies de singularisation. L'évolution du livre IV du commentaire commun des Sentences de Vienne, in: Monica BRînZEI (Hg.), Nicholas of Dinkelsbühl and the Sentences at Vienna in the Early Fifteenth Century (Studia Sententiarum 1), Turnhout 2015, S. 85-267, hier S. 121. 
fenden Grundbestimmungen der Ähnlichkeit, der Repräsentation und des wie auch immer gearteten Effekts nicht mehr zu rütteln war. Insofern erstaunt es nicht, dass in der traditionalistischen Wende der Scholastik im Übergang vom 14. zum 15. Jahrhundert das ,Mitwirkungs-Modell‘ wieder salonfähig wurde und die Kritik an einer verkürzten Lesart des augustinischen Bildes weitgehend in Vergessenheit geriet: ${ }^{47}$ Es stimmte dieses Bild inzwischen einfach zu gut mit dem allgemeinen Verständnis dessen überein, was ein Sakrament sei, denn schließlich hatte es dieses Verständnis ja auch grundlegend geprägt.

Einer der klarsten Vertreter einer intrinsischen sakramentalen Wirkweise war am Beginn des 15. Jahrhunderts kein geringerer als Jan Hus höchstpersönlich, der in der klassischen Terminologie des Thomas von Aquin die Sakramente für instrumentale Ursachen der Gnade hielt. ${ }^{48}$ Tatsächlich erweisen sich bei genauerer Betrachtung nun sowohl der Hussit Peter Payne als auch der Dominikaner Torquemada als Verteidiger der Wassersymbolik im Sakramentenverständnis: Denn Peter Payne weitete das augustinische Bild der Wasserwirkung so konsequent auf die Eucharistie aus, dass er auch den eucharistischen Elementen eine vom Taufwasser inspirierte Wirksamkeit zusprechen wollte und entsprechend nicht hinnehmen konnte, dass einem Großteil der Gläubigen eines dieser Elemente und damit dessen Wirksamkeit entzogen blieb. In der scholastisch etablierten Wassersymbolik hingegen ging dies zu weit. Juan de Torquemada verteidigte implizit zwar die Allgemeingültigkeit des Wasserbildes, das sich auch auf das Weihwasser ausweiten ließ, so dass auch diesem ein eigener, intrinsischer Effekt zugesprochen werden könne, doch behielt er die scholastische Reserve einem allzu direkten, kausalen Einbezug der sakramentalen Elemente gegenüber bei, wie sie sich letztlich schon bei Augustinus und seiner ursprünglichen Formulierung des Wasserbildes gefunden hatte: Wie das äußerlich reinigende Wasser bloß repräsentierendes Instrument der innerlich reinigenden Gnade war, so waren auch die eucharistischen Elemente nur äußerlicher Anschein, deren innerer Effekt daher sub utraque specie ein- und derselbe blieb. Wo der eine auf die Wirksamkeit setzte, betonte der andere die Ähnlichkeit - und doch ging historisch gesehen beides, wenn nicht auf die so große Kraft im Wasser, so doch auf die Symbolkraft des Wassers zurück.

47 Dazu ZAHnd (Anm. 14), S. 374-378.

48 Jan Hus, Super IV Sententiarum, IV d. 1, hrsg. v. Wenzel FlaišHans u. Marie KomínKová, Prag 1904, S. 514. 